\title{
An Efficient Chip-level Time Slack Allocation Algorithm for Dual-Vdd FPGA Power Reduction.
}

\author{
Yan Lin ${ }^{1}$, Yu Hu ${ }^{1}$, Lei He ${ }^{1}$ and Vijay Raghunat ${ }^{2}$ \\ Electrical Engineering Dept., UCLA, Los Angeles, CA ${ }^{1}$ \\ Purdue University, La Fayette, IN ${ }^{2}$
}

\begin{abstract}
To reduce FPGA power, a linear programming (LP) based time slack allocation algorithm, EdTLC-LP, has been proposed recently for $\mathrm{Vdd}$-programmable interconnects without using Vdd-level converters for mixed wire lengths. However, it takes a long time to solve the LP problem for time slack allocation. In this paper, we develop EdTLC-NW, a slack allocation algorithm based on min-cost network flow to reduce runtime. Compared to single Vdd FPGA with power-gating, EdTLC-LP and EdTLC-NW reduce interconnect power by $52.71 \%$ and $52.52 \%$, respectively. EdTLC-NW achieves as good results as EdTLC-LP but runs $8 X$ faster on average. Furthermore, the speedup increases for larger circuits and EdTLC-NW is $20 X$ faster for the largest circuit.

Categories and Subject Descriptors: B.7.2 [Integrated Circuits]: Design aids

General Terms: Algorithms, Design

Keywords: Low power, time slack, FPGA
\end{abstract}

\section{INTRODUCTION}

FPGA power modeling and reduction has become an active research area recently. $[1,2]$ present power evaluation frameworks for generic parameterized FPGA architectures, and show that both interconnect and leakage power are significant for nanometer FPGAs. [3] studies the interaction of a suite of power-aware FPGA CAD algorithms without changing the existing FPGAs. [4] proposes a configuration inversion method to reduce leakage power of multiplexers. In addition, dual-Vdd and Vdd programmability have been applied to FPGA to reduce power. $[5,6]$ are the first work introducing dual-Vdd and field programmability of Vdd to FPGA. Vdd programmability has been applied to both FPGA logic blocks [5, 6] and interconnects [7, 8, 9].

A Vdd-level converter is needed when a low-Vdd (VddL) circuit elements drives a high-Vdd $(\mathrm{VddH})$ circuit element to avoid excessive leakage. [8] inserts a level converter in front of each interconnect switch to provide the fine-grained Vdd programmability for interconnects. However, it has

\footnotetext{
${ }^{*}$ This paper is partially supported by NSF grant CCR-0306682. Address comments to lhe@ee.ucla.edu.

Permission to make digital or hard copies of all or part of this work for personal or classroom use is granted without fee provided that copies are not made or distributed for profit or commercial advantage and that copies bear this notice and the full citation on the first page. To copy otherwise, to republish, to post on servers or to redistribute to lists, requires prior specific permission and/or a fee.

ISLPED'06, October 4-6, 2006, Tegernsee, Germany.

Copyright 2006 ACM 1-59593-462-6/06/0010 ...\$5.00.
}

been shown in [10] that this fine-grained Vdd-level converter insertion may introduce large leakage and area overhead. Recently, a few approaches have been presented without directly using level converters in Vdd-programmable interconnects. [9] uses the positive feedback PMOS transistor in the level-restore buffer as an alternative level converter with much reduced area and power overhead. [7] enforces that all the routing trees driven by (driving) a logic block have the same Vdd-level as the source (sink) logic block when level converters are inserted at CLB inputs (outputs). [11] uses a smaller granularity, a routing tree, as the unit in Vdd-level assignment. [10] further allows a mix of Vddlevels within a routing tree, but only VddH switches can drive VddL switches. [12] extends the algorithms in [10] for mixed interconnect wire lengths.

In this paper, we use the same circuit design from $[10,12]$ and aim to improve the linear programming (LP) based time slack allocation algorithm, EdTLC-LP. In EdTLC-LP, time slack is allocated to each routing tree by formulating the problem as an LP problem to minimize power. However, it takes unacceptable runtime for EdTLC-LP to solve the LP problem for time slack allocation (more than 10 hours for the largest circuit clma on a $1.9 \mathrm{GHz}$ Xeon machine). Our contribution is to formulate the time slack allocation problem as a min-cost network flow problem and present a new algorithm, $E d T L C-N W$, which significantly reduces the run-time. Using single-Vdd FPGA with power-gating as the baseline, EdTLC-LP and EdTLC-NW reduce interconnect power by $52.71 \%$ and $52.52 \%$, respectively. EdTLC-NW achieves as good results as EdTLC-LP but runs $8 X$ faster on average. Furthermore, the speedup increases for larger circuits and EdTLC-NW achieves up to $20 X$ speedup in overall runtime.

The rest of the paper is organized as follows. Section 2 introduces background and modeling. Section 3 reviews the LP based budgeting. Section 4 describes netflow based budgeting for interconnects with mixed wire lengths. Section 5 discusses the experimental results. Section 6 concludes this paper.

\section{PRELIMINARIES}

\subsection{Delay and Power Modeling with Dual-Vdd}

To make the presentation simple, we summarize the notations frequently used in this paper in Table 1. They will be explained in detail when first used.

A directed acyclic timing graph $\mathcal{G}(\mathcal{V}, \mathcal{E})$ [13] is constructed to model the circuit for timing analysis. The Elmore delay model is used to calculate the routing delay. We define the 


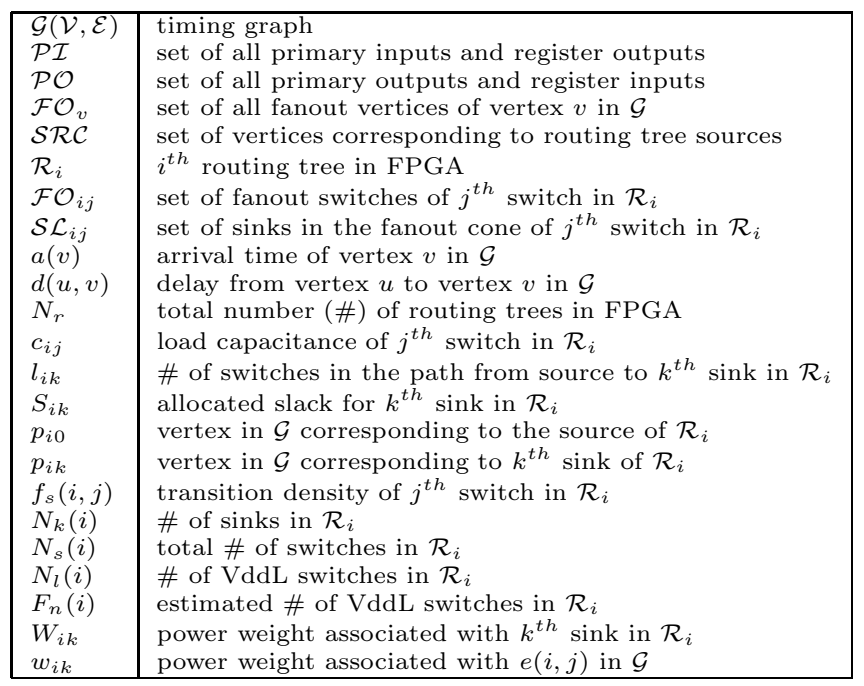

Table 1: Notations frequently used in this paper

fanout cone of a switch as the sub-tree of the routing tree rooted at the switch. Dynamic power occurs when a signal transition happens at the gate output. Although timing change may change the transition density, we assume that the transition density for an interconnect switch will not change when VddL is used. Let $v_{i j}$ indicate Vdd-level of $j^{\text {th }}$ switch in $\mathcal{R}_{i}$ as follows

$$
v_{i j}= \begin{cases}1 & \text { if Vdd-level of } j^{t h} \text { switch in } \mathcal{R}_{i} \text { is VddH } \\ 0 & \text { if Vdd-level of } j^{t h} \text { switch in } \mathcal{R}_{i} \text { is VddL }\end{cases}
$$

The interconnect power reduction $P_{r}$ using programmable dual-Vdd can be expressed as

$$
P_{r}=\sum_{i=0}^{N_{r}-1} \sum_{j=0}^{N_{s}(i)-1}\left(1-v_{i j}\right)\left(0.5 f_{c l k} f_{s}(i, j) c_{i j} \Delta V d d^{2}+\Delta P_{s}(i, j)\right)
$$

which is the sum of dynamic and leakage power reduction. $N_{r}$ is the total number of routing trees, $f_{s}(i, j)$ is the transition density of $j^{\text {th }}$ switch in $i^{\text {th }}$ routing tree $\mathcal{R}_{i}, N_{s}(i)$ is the number of switches in $\mathcal{R}_{i}$, and $\Delta P_{s}(i, j)$ and $c_{i j}$ are the leakage power reduction and load capacitance of each switch, respectively.

Dual-Vdd tree based level converter insertion [10,12] is used in this paper. A mix of Vdd-levels within one routing tree is allowed, and the Vdd-level constraints are

$$
v_{i k} \leq v_{i j} \quad 0 \leq i<N_{r} \wedge 0 \leq j<N_{s}(i) \wedge k \in \mathcal{F} \mathcal{O}_{i j}
$$

i.e., no VddL switch should drive VddH switches. $\mathcal{F O}_{i j}$ gives the set of fanout switches of $j^{\text {th }}$ switch in $\mathcal{R}_{i}$.

\section{LINEAR PROGRAMMING BASED BUDGETING}

In this section, we review the LP based time slack allocation algorithm, $E d T L C-L P$, for mixed interconnect wire lengths [12]. Time slack is first allocated to each routing tree by formulating the problem as an LP problem considering the load capacitance of each switch explicitly. A bottom-up assignment algorithm is then performed to achieve the optimal solution within each routing tree for the allocated time slack. A refinement step is finally performed to leverage surplus time slack.

\subsection{Estimation of Interconnect Power Reduction}

Estimating power reduction given the allocated slack is the key for the LP and netflow based algorithms. There is an upper bound for slack, which is the delay increase when VddL is assigned to all the switches in a tree. Clearly, slack more than the upper bound cannot lead to more VddL switches. The slack upper bound constraints can be expressed as

$$
0 \leq S_{i k} \leq D_{i k} \quad 0 \leq i<N_{r} \wedge 1 \leq k \leq N_{k}(i)
$$

where $N_{k}(i)$ is the number of sinks in $\mathcal{R}_{i}$ and $D_{i k}$ is the delay increase of the path from the source to $k^{\text {th }} \operatorname{sink}$ in $\mathcal{R}_{i}$ when VddL is assigned to all the switches in that path.

Let $l_{i k}$ represent the number of switches in the path from the source to the $k^{t h} \operatorname{sink}$ in $\mathcal{R}_{i}$. Slack $S_{i k}$ is first transformed into $s_{i k}$, which is expressed in number of switches as follows,

$$
s_{i k}=\frac{S_{i k}}{D_{i k}} \cdot l_{i k}
$$

Let $c_{i j}$ represent the load capacitance of the $j^{\text {th }}$ switch in $\mathcal{R}_{i}$. Let $C_{i k}$ represent the total load capacitance of the switches in the path from the source to the $k^{t h} \operatorname{sink}$ in $\mathcal{R}_{i}$. Sink list $\mathcal{S} \mathcal{L}_{i j}$ is defined as the set of sinks in the fanout cone of the $j^{\text {th }}$ switch in $\mathcal{R}_{i}$. The number of VddL switches given the allocated slack is then estimated as

$$
F_{n}(i)=\sum_{j=0}^{N_{s}(i)-1} \min \left(\frac{s_{i k}}{C_{i k}} \cdot c_{i j}: \forall k \in \mathcal{S} \mathcal{L}_{i j}\right)
$$

The rationale is that we consider $k^{t h}$ sink with minimum $s_{i k} c_{i j} / C_{i k}$ in sink list $\mathcal{S} \mathcal{L}_{i j}$ as the most critical sink to $j^{\text {th }}$ switch in $\mathcal{R}_{i}$.

The dynamic/leakage power reduction of the tree $\mathcal{R}_{i}$ is estimated as the sum of the dynamic/leakage power reduction of each switch in $\mathcal{R}_{i}$ and can be expressed as,

$$
\begin{gathered}
P_{d r}(i)=0.5 f_{c l k} \cdot \Delta V d d^{2} \sum_{j=0}^{N_{S}(i)-1}\left[\min \left(\frac{s_{i k}}{C_{i k}} \cdot c_{i j}: \forall k \in \mathcal{S} \mathcal{L}_{i j}\right) \cdot f_{\mathcal{S}}(i, j) \cdot c_{i j}\right] \\
P_{l r}(i)=\sum_{j=0}^{N_{S}(i)-1}\left[\min \left(\frac{s_{i k}}{C_{i k}} \cdot c_{i j}: \forall k \in \mathcal{S} \mathcal{L}_{i j}\right) \cdot \Delta P_{S}(i, j)\right]
\end{gathered}
$$

where $\Delta P_{s}(i, j)$ is the leakage power difference of $j^{t h}$ switch in $\mathcal{R}_{i}$ between $\mathrm{VddH}$ and $\mathrm{VddL}$. Wire segments with different lengths might be driven by switches with different sizes.

\subsection{LP Problem Formulation}

Similar to [10], the net-based formulation is used, which partitions the constraints on path delay into constraints on delay across circuit elements or routing. Let $a(v)$ be the arrival time for vertex $v$ in $\mathcal{G}$ and the timing constraints become

$$
\begin{array}{cl}
a(v) \leq T_{\text {spec }} & \forall v \in \mathcal{P O} \\
a(v)=0 & \forall v \in \mathcal{P I} \\
a(u)+d(u, v) \leq a(v) & \forall u \in \mathcal{V} \wedge v \in \mathcal{F O}_{u}
\end{array}
$$

where $\mathcal{V}$ is the set of vertices in $\mathcal{G}, d(u, v)$ is the delay from vertex $u$ to $v$ and $\mathcal{F} \mathcal{O}_{u}$ is the set of fanout vertices of $u$.

The objective is to maximize interconnect power reduction given by the sum of (6) and (7). To incorporate them into mathematical programming, we introduce a variable $f_{n}(i, j)$ for $j^{\text {th }}$ switch in $\mathcal{R}_{i}$ and some additional constraints. The 
new objective function after transformation plus the additional constraints can be expressed as

$$
\begin{array}{r}
\text { Maximize } \sum_{i=0}^{N_{r}-1} 0.5 f_{c l k} \Delta V d d^{2} \sum_{j=0}^{N_{s}(i)-1} f_{n}(i, j) f_{s}(i, j) c_{i j} \\
+\sum_{i=0}^{N_{r}-1} \sum_{j=0}^{N_{s}(i)-1} f_{n}(i, j) \Delta P_{s}(i, j)
\end{array}
$$

s.t.

$$
f_{n}(i, j) \leq \frac{s_{i k}}{C_{i k}} c_{i j} \quad 0 \leq i<N_{r} \wedge 0 \leq j<N_{s}(i) \wedge \forall k \in \mathcal{S} \mathcal{L}_{i j}
$$

The timing constraints (10) is then modified as follows. For the edges corresponding to routing in $\mathcal{G}$, the constraints considering slack can be expressed as

$$
\begin{gathered}
a\left(p_{i 0}\right)+d\left(p_{i 0}, p_{i k}\right)+S_{i k} \leq a\left(p_{i k}\right) \\
0 \leq i<N_{r} \wedge \forall p_{i k} \in \mathcal{F} \mathcal{O}_{p_{i 0}}
\end{gathered}
$$

where vertex $p_{i 0}$ is the source of $\mathcal{R}_{i}$ in $\mathcal{G}$, vertex $p_{i k}$ is $k^{t h}$ sink of $\mathcal{R}_{i}$ in $\mathcal{G}, S_{i k}$ is the slack allocated to $k^{t h}$ sink in $\mathcal{R}_{i}$ and $d\left(p_{i 0}, p_{i k}\right)$ is the delay from $p_{i 0}$ to $p_{i k}$ in $\mathcal{R}_{i}$ using VddH. For the edges other than routing in $\mathcal{G}$, the constraints can be expressed as

$$
a(u)+d(u, v) \leq a(v) \quad \forall u \in \mathcal{V} \wedge u \notin \mathcal{S R C} \wedge v \in \mathcal{F O}_{u}
$$

where $\mathcal{S R C}$ contains vertices corresponding to routing tree sources.

The time slack allocation problem is formulated using objective function (11), additional constraints (12), slack upper bound constraints (3), and timing constraints (8), (9), (13) and (14). It is easy to verify that all the constraints are linear, and the objective function (11) is also linear.

THEOREM 1. The time slack allocation problem is a linear programming (LP) problem.

\section{NETWORK FLOW BASED BUDGETING}

The runtime of time slack allocation in EdTLC-LP can be very long for large circuits mainly due to the expensive computational time of linear programming. In this section, we formulate the time slack allocation problem as a min-cost network flow problem and present a new algorithm, EdTLC$N W$, with significantly reduced runtime.Similar network flow formulation has been used for timing budgeting in high level synthesis [14].

\subsection{Network Flow Formulation}

We first deliberately distribute slack $s_{i k}$ to the switches in the path from the source to to $k^{t h}$ sink in $\mathcal{R}_{i}$. As the min function in (5) cannot be efficiently handled by a network flow formulation, we define the sink with the minimum slack as the critical sink in the fanout cone of a switch with multiple sinks. We then use $s_{i k} c_{i j} / C_{i k}$ of this critical sink to replace the min operator over all sinks in the fanout cone of a switch. The dynamic power reduction (6) and leakage power reduction (7) for $\mathcal{R}_{i}$ can be rewritten as follows,

$$
\begin{gathered}
P_{d r}(i)=0.5 f_{c l k} \cdot \Delta V d d^{2} \cdot \sum_{j=0}^{N_{s}(i)-1} S_{i k} \cdot\left[f_{s}(i, j) \cdot \frac{l_{i k} \cdot c_{i j}^{2}}{D_{i k} \cdot C_{i k}}\right] \\
P_{l r}(i)=\sum_{j=0}^{N_{s}(i)-1} S_{i k} \cdot\left[\left(\frac{l_{i k} \cdot c_{i j}}{D_{i k} \cdot C_{i k}}\right) \cdot \Delta P_{s}(i, j)\right]
\end{gathered}
$$

The objective function can be rewritten as following by merging the coefficient of the slack $S_{i k}$ of $k^{t h} \operatorname{sink}$ in $\mathcal{R}_{i}$ as follows,

$$
\begin{gathered}
\text { Maximize } \sum_{i=0}^{N_{r}-1} \sum_{k=0}^{N_{k}(i)-1} W_{i k} \cdot S_{i k}=\sum_{\forall S i n k} W_{i k} \cdot S_{i k} \\
W_{i k}=\sum_{\forall j \in \mathcal{U} \mathcal{B C}_{i k}}\left[0.5 f_{c l k} \Delta V d d^{2} c_{i j} f_{s}(i, j)+\Delta P_{s}(i, j)\right] \cdot \frac{c_{i j} \cdot D_{i k}}{\left(C_{i k} \cdot l_{i k}\right)}
\end{gathered}
$$

where set $\mathcal{U B C}_{i k}$ include all switches with $k^{t h}$ sink as the critical sink in $\mathcal{R}_{i}$.

Since $W_{i k}>0$ for all sinks, we can restrict timing constraint (13) as the following equation to maximize the objective function,

$S_{i k}=a\left(p_{i k}\right)-a\left(p_{i 0}\right)-d\left(p_{i 0}, p_{i k}\right), \quad 0 \leq i<N_{r} \wedge \forall p_{i k} \in \mathcal{F} \mathcal{O}_{p_{i 0}}$

After substituting $S_{i k}$ using (19) and rearrangement, objective function (17) can be expressed as,

Maximize $\quad \sum_{i=0}^{N_{r}-1} \sum_{k=0}^{N_{k}(i)-1} W_{i k} \cdot\left[a\left(p_{i k}\right)-a\left(p_{i 0}\right)-d\left(p_{i 0}, p_{i k}\right)\right]$

Similarly, slack bound constraint (3) can be rewritten as

$$
\begin{array}{r}
a\left(p_{i 0}\right)-a\left(p_{i k}\right) \leq-d\left(p_{i 0}, p_{i k}\right) \\
a\left(p_{i k}\right)-a\left(p_{i 0}\right) \leq d\left(p_{i 0}, p_{i k}\right)+D_{i k}
\end{array}
$$

We then merge the timing constraint (21) and (14) into the general expression (10).

Similar to [14], a virtual input node $(S I)$ and a virtual output node $(S O)$ are added into $\mathcal{G}$ to connect all nodes in $\mathcal{P} \mathcal{I}$ and $\mathcal{P O}$, respectively. All edges connected to $S I$ and $S O$ have zero delay. We add a backward edge $e\left(p_{i k}, p_{i 0}\right)$ for each source sink pair in $\mathcal{R}_{i}$. A delay of $-\left(d\left(p_{i 0}, p_{i k}\right)+\right.$ $\left.D_{i k}\right)$ is associated to $e\left(p_{i k}, p_{i 0}\right)$ to represent the slack upper bound. A virtual edge $e(S O, S I)$ with delay $-T_{\text {spec }}$ is then added. All constraints can now be represented by edges in $\mathcal{G}$. For example, edge $e(u, v)$ with delay $d(u, v)$ represents constraint $a(u)-a(v)<-d(u, v)$.

To represent the objective function $(20)$ in $\mathcal{G}$, we associate a weight $w_{u v}$ in each edge $e(u, v)$. For those edges $e\left(p_{i 0}, p_{i k}\right)$ corresponding to routing, let $w_{p_{i 0} p_{i k}}=W_{i k}$. For other edges, let $w_{u v}=0$. The objective function (20) can then be rewritten as,

$$
\begin{gathered}
\text { Maximize } \sum_{v \in V} a(v)\left(\sum_{u \in \mathcal{F} \mathcal{I}_{v}} w_{u v}-\sum_{u \in \mathcal{F} \mathcal{O}_{v}} w_{v u}\right) \\
-\sum_{i=0}^{N_{r}-1} \sum_{k=0}^{N_{k}(i)-1} d\left(p_{i 0}, p_{i k}\right)
\end{gathered}
$$

where $\sum_{i=0}^{N_{r}-1} \sum_{k=0}^{N_{k}(i)-1} d\left(p_{i 0}, p_{i k}\right)$ is a constant and can be removed from the objective function $(23)$, and $\mathcal{F} \mathcal{I}_{v} / \mathcal{F} \mathcal{O}_{v}$ is fanin/fanout set of vertex $v$.

For the optimization problem with constraints (10) and (22) and objective function (23), its dual problem is

$$
\begin{gathered}
\min \quad \sum_{\left.e_{(} i, j\right) \in \mathcal{E}}\left(d(i, j)+D_{i j}\right) \cdot z_{i j}-d(i, j) \cdot y_{i j} \\
\text { s.t. } \quad \sum_{e(k, i) \in \mathcal{E}}\left(y_{k i}-z_{k i}\right)-\sum_{e(i, j) \in \mathcal{E}}\left(y_{i j}-z_{i j}\right)=\rho_{i} \\
\rho_{i}=\sum_{j \in \mathcal{F} \mathcal{I}_{i}} w_{j i}-\sum_{k \in \mathcal{F} \mathcal{O}_{k}} w_{i k} \\
y_{i j}, z_{i j} \in R_{+}
\end{gathered}
$$


To verify that the above dual problem is a min-cost network flow problem on $\mathcal{G}, y_{i j}$ is the flow along $e(i, j)$ with cost $-d(i, j), z_{i j}$ is the flow along $e(j, i)$, which corresponds to routing and is associated with cost $d(i, j)+D_{i j}$. Obviously, no negative cycle is introduced by the backward edges. $\rho_{i}$ is the demand in each vertex. Note that $\sum_{i \in \mathcal{V}} \rho_{i}=0$ is satisfied as required in the min-cost network flow problem. Hence, we have the following theorem.

THEOREM 2. The dual problem of the time slack allocation problem is a min-cost network flow problem.

After solving the min-cost network flow problem, we can get the solutions for variables $y_{i j}$ and $z_{i j}$. Similar to [14], we can calculate the solution of the primal problem. We first construct the residual graph $\mathcal{G}^{\prime}\left(\mathcal{V}, \mathcal{E}^{\prime}\right)$ from the original $\mathcal{G}$. For any edge $e(i, j)$ in $\mathcal{G}^{\prime}$ with non-zero flow, there are two edges $e(j, i)$ and $e(i, j)$ in $\mathcal{G}^{\prime}$. The cost of each backward edge $e(j, i)$ is $d(i, j)$, and is equal to the complement of the forward edge cost. Let $\delta_{i}$ be the shortest distance from $S I$ to vertex $i$ in $\mathcal{G}^{\prime}$. It has been proved in [14] that $a(i)=-\delta_{i}$ is an optimal solution to the primal problem. We use the push-relabel algorithm [15] for min-cost flow problem and Bellman-Ford algorithm [16] for shortest path problem.

\subsection{Comparison Between EdTLC-LP and EdTLC-NW}

The basic difference between EdTLC-LP and EdTLC-NW is the way to calculate the slack for each switch. EdTLCLP chooses the minimum $s_{i k} c_{i j} / C_{i k}$ among all sinks while EdTLC-NW chooses the $s_{i k} c_{i j} / C_{i k}$ of the critical sink. Let $k^{\text {th }}$ sink be the critical sink in the fanout cone of $j^{t h}$ switch in $\mathcal{R}_{i}$. EdTLC-NW estimates number of VddL switches in $\mathcal{R}_{i}$ as

$$
F_{n}(i)=\sum_{j=0}^{N_{s}(i)-1} \frac{s_{i k}}{C_{i k}} \cdot c_{i j}
$$

It has been proved in [10] that (5) can always give a lower bound of the number of VddL switches that can be achieved in $\mathcal{R}_{i}$ for uniform wire length. However, (5) cannot always give an infimum (the greatest lower bound) for the VddL switch number. Figure 1 (a) shows a simple example (uniform length of wire segments is assumed for simplicity).
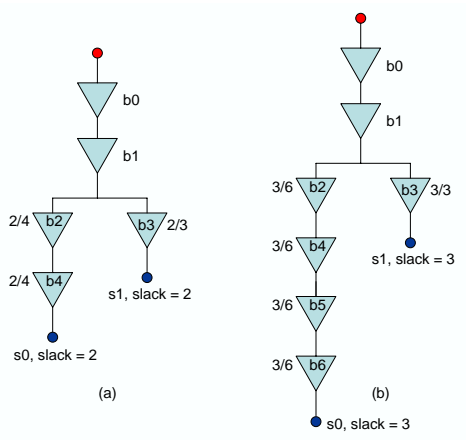

Figure 1: An example of estimated VddL switch \#

Suppose $S 1$ is the critical sink based on the current timing graph, and a slack of 2 is allocated to both $S 0$ and $S 1$. (5) estimates VddL switch number as $(2 / 4+2 / 4+$
$2 / 3+2 \min (2 / 4+2 / 3))=8 / 3$ while $(28)$ gives an estimation as $(2 / 4+2 / 4+2 / 3+2 \cdot 2 / 3)=3$. Obviously, we CAN achieve three VddL switches while satisfying the allocated slack $S 0=S 1=2$. This indicates that EdTLC-LP cannot always give the infimum of VddL switch number.

On the other hand, EdTLC-NW always gives a greater estimation than EdTLC-LP while it might give an overestimated result. As shown in Figure 1 (b), suppose $S 1$ is still the critical sink. The slacks allocated to $S 0$ and $S 1$ are both 3 . (5) gives an estimation as $(3 / 6+3 / 6+$ $3 / 6+3 / 6+3 / 3+2 \min (3 / 6+3 / 3))=4$ while $(28)$ gives $(3 / 6+3 / 6+3 / 6+3 / 6+3 / 3+2 \cdot 3 / 3)=5$. However, we cannot achieve five VddL switches while satisfying the allocated slacks $S 0=S 1=3$. This indicates that EdTLC-NW may overestimate the VddL switch number in some cases.

In summary, EdTLC-LP may give a conservative estimation of power savings while EdTLC-NW may give an overoptimistic estimation. In the experiments to be presented in Section 5, the two formulations give a similar estimation for most cases due to the fact that the most critical sink is usually the one with the minimum $s_{i k} c_{i j} / C_{i k}$. In addition, EdTLC-NW can achieve results close to EdTLC-LP in terms of power reduction but with significantly shorter runtime.

\section{EXPERIMENTAL RESULTS 5.1 Experimental Settings}

We conduct the experiments on the largest MCNC benchmarks [17] including ten combinational circuits (group I in Table 2) and ten sequential circuits (group II in Table 2). We map them into FPGA with LUT size of 4 and cluster size of 10 . We use the same Vdd-programmable logic blocks and interconnects in [10], but with a mix of different interconnect wire lengths. We use $60 \%$ length 4 wire and $40 \%$ length 8 wire for better performance and area tradeoff, as suggested in [18]. The unused interconnect switches are power-gated in all cases. Similar to [8], we customize the FPGA chip size for each benchmark circuit and use the smallest chip that fits each benchmark. We use $1.3 \mathrm{v}$ for $\mathrm{VddH}$ and $0.8 \mathrm{v}$ for VddL same as [10] in our experiments at 100 $\mathrm{nm}$ technology node. We perform dual-Vdd assignment without delay increase compared to the circuit using only $\mathrm{VddH}$ in the rest of the paper.

We first use VPR [13] for single-Vdd placement and routing. Before applying budgeting algorithms to the Vdd programmable interconnects, a sensitivity based assignment [6] is first performed to assign Vdd-level for Vdd-programmable logic blocks without performance loss ${ }^{1}$. The cycle-accurate FPGA power simulator fpgaEva-LP2 [11] is then used to calculate power.

\subsection{Comparison of Interconnect Power}

We first compare the the number of VddL switches achieved by EdTLC-LP and EdTLC-NW in Table 2, as it is a good indication of power reduction. The number of VddL switches is expressed in percent of used switch number. EdTLC-LP and EdTLC-NW achieve $84.43 \%$ and $84.05 \%$ VddL switches, respectively. Both achieve almost the same number of VddL switches.

\footnotetext{
${ }^{1}$ Both algorithms EdTLC-LP and EdTLC-NW do consider the fact that VddL logic blocks consume time slack.
} 
We then present the interconnect power reduction achieved by EdTLC-LP and EdTLC-NW in Table 3. Single-Vdd FPGA with power-gating is used as the baseline for interconnect power. Columns 2-3 present the interconnect dynamic and leakage power for the baseline case. Columns 4-5 present the overall interconnect power reduction for EdTLC-LP and EdTLC-NW . Compared to the baseline case, EdTLC-LP and EdTLC-NW reduce total interconnect power by $52.71 \%$ and $52.03 \%$, respectively. We also present the interconnect dynamic power and leakage power reduction in columns 69. Compared to the baseline, EdTLC-LP and EdTLC-NW reduce dynamic power by $52.03 \%$ and $51.69 \%$, and leakage power by $62.71 \%$ and $62.51 \%$, respectivly. Clearly, EdTLCNW achieves as good results as EdTLC-LP.

For both algorithms, we also present the contribution of refinement step in Table 2 and Table 3. The refinement step achieves $3.49 \%$ and $4.17 \%$ VddL switches for EdTLC-LP and EdTLC-NW , respectively. Compared to baseline, the refinement step in EdTLC-LP /EdTLC-NW obtains 3.35\%/ $1.03 \%$ more power reduction, $3.45 \% / 0.96 \%$ more dynamic power reduction and $2.23 \% / 2.57 \%$ more leakage power reduction, respectively. It is clear that the refinement step is effective to distribute surplus time slack and further reduce interconnect power. The first source of the surplus time slack is the difference between the continuous problem formulations (i.e., the allocated slack is continuous in budgeting) and the fact that Vdd-level assignment is discrete (i.e., the slack consumed by a $\mathrm{VddL}$ switches must be $\Delta d$ ). Secondly, the objective of our formulations is to maximize the estimated power reduction instead of the exact power reduction. The two may be different due to the fact that not all allocated slack is useful for power reduction.

\begin{tabular}{|c|c|c|c|c|}
\hline & Circuit & Cluster\# & EdTLC-LP & EdTLC-NW \\
\hline \multirow{6}{*}{ I } & ex5p & 123 & $68.32 \%(3.43 \%)$ & $67.99 \%(4.87 \%)$ \\
& apex4 & 134 & $75.38 \%(6.65 \%)$ & $73.27 \%(8.04 \%)$ \\
& misex3 & 153 & $73.77 \%(4.12 \%)$ & $72.41 \%(9.11 \%)$ \\
& alu4 & 162 & $78.67 \%(4.89 \%)$ & $78.08 \%(6.54 \%)$ \\
& seq & 198 & $70.49 \%(4.93 \%)$ & $69.52 \%(6.63 \%)$ \\
& apex2 & 213 & $78.48 \%(3.98 \%)$ & $78.09 \%(4.74 \%)$ \\
& des & 218 & $82.13 \%(1.94 \%)$ & $82.01 \%(1.86 \%)$ \\
& spla & 399 & $78.46 \%(4.40 \%)$ & $78.36 \%(6.01 \%)$ \\
& ex1010 & 493 & $78.77 \%(5.55 \%)$ & $78.59 \%(6.84 \%)$ \\
& pdc & 568 & $79.76 \%(4.19 \%)$ & $79.29 \%(5.16 \%)$ \\
\hline \multirow{6}{*}{ II } & tseng & 131 & $97.02 \%(2.58 \%)$ & $97.07 \%(0.71 \%)$ \\
& dsip & 162 & $91.70 \%(1.10 \%)$ & $91.67 \%(1.67 \%)$ \\
& diffeq & 195 & $92.93 \%(2.58 \%)$ & $92.90 \%(1.21 \%)$ \\
& s298 & 256 & $89.62 \%(2.93 \%)$ & $89.59 \%(1.41 \%)$ \\
& bigkey & 294 & $81.43 \%(2.18 \%)$ & $80.88 \%(7.27 \%)$ \\
& elliptic & 421 & $98.72 \%(1.92 \%)$ & $98.75 \%(0.28 \%)$ \\
& frisc & 595 & $99.19 \%(4.74 \%)$ & $99.19 \%(2.58 \%)$ \\
& s38584.1 & 704 & $97.77 \%(1.86 \%)$ & $97.75 \%(0.65 \%)$ \\
& s38417 & 847 & $90.15 \%(2.53 \%)$ & $89.86 \%(2.93 \%)$ \\
& clma & 1358 & $85.93 \%(3.20 \%)$ & $85.66 \%(3.38 \%)$ \\
\hline & Ave & 266 & $84.43 \%(3.49 \%)$ & $84.05 \%(4.17 \%)$ \\
\hline
\end{tabular}

Table 2: Percentage of VddL switches achieved by EdTLC-LP and EdTLC-NW

\subsection{Comparison of Runtime}

Table 4 compares the runtime of EdTLC-LP and EdTLCNW. Column "budget" refers to the runtime for distributing slack to each tree. The simplex method based LP solver [19] is used in EdTLC-LP . Column "total" presents the overall runtime. Column "speedup" shows the ratio of speedup achieved by EdTLC-NW compared to EdTLC-LP. EdTLCNW achieves as good results as EdTLC-LP but runs $8 X$ faster on average. It is clear that the speedup of the bud- geting time increases for the larger circuits. For the largest circuit clma, which contains over 1k clusters, EdTLC-NW achieves up to $6000 X$ speedup in time slack allocation and $20 X$ speedup in overall runtime. The current commercial FPGA designs often contain 10k to 100k clusters [20], which indicates that the EdTLC-NW may have more speedup than that reported in this paper in practice. The min-cost network flow based algorithm EdTLC-NW takes negligible runtime in time slack allocation compared to EdTLC-LP . Clearly, the efficiency of EdTLC-NW makes our algorithm highly scalable, especially for the applications that need iterative budgeting for different time specifications.

\begin{tabular}{|c|c|c|c|c|c|c|}
\hline \multirow{2}{*}{ cir } & \multicolumn{2}{|c|}{ EdTLC-NW } & \multicolumn{2}{c|}{ EdTLC-LP } & \multicolumn{2}{c|}{ speedup(X) } \\
\cline { 2 - 7 } & budget & total & budget & total & budget & total \\
\hline ex5p & 1 & 33 & 1187 & 1254 & $118 \mathrm{x}$ & $5 \mathrm{x}$ \\
apex4 & 1 & 33 & 184 & 228 & $184 \mathrm{x}$ & $7 \mathrm{x}$ \\
misex3 & 1 & 38 & 155 & 227 & $155 \mathrm{x}$ & $6 \mathrm{x}$ \\
alu4 & 1 & 36 & 101 & 131 & $101 \mathrm{x}$ & $4 \mathrm{x}$ \\
seq & 1 & 55 & 179 & 216 & $179 \mathrm{x}$ & $4 \mathrm{x}$ \\
apex2 & 1 & 78 & 413 & 502 & $413 \mathrm{x}$ & $6 \mathrm{x}$ \\
des & 1 & 95 & 327 & 444 & $327 \mathrm{x}$ & $5 \mathrm{x}$ \\
spla & 1 & 300 & 837 & 1181 & $837 \mathrm{x}$ & $4 \mathrm{x}$ \\
ex1010 & 2 & 346 & 2391 & 2804 & $1196 \mathrm{x}$ & $8 \mathrm{x}$ \\
pdc & 2 & 803 & 3633 & 4496 & $1817 \mathrm{x}$ & $6 \mathrm{x}$ \\
\hline tseng & 1 & 32 & 83 & 91 & $83 \mathrm{x}$ & $3 \mathrm{x}$ \\
dsip & 1 & 44 & 181 & 223 & $181 \mathrm{x}$ & $5 \mathrm{x}$ \\
diffeq & 1 & 41 & 252 & 321 & $252 \mathrm{x}$ & $8 \mathrm{x}$ \\
s298 & 1 & 96 & 371 & 494 & $371 \mathrm{x}$ & $5 \mathrm{x}$ \\
bigkey & 1 & 89 & 478 & 589 & $478 \mathrm{x}$ & $7 \mathrm{x}$ \\
elliptic & 1 & 229 & 877 & 1128 & $877 \mathrm{x}$ & $5 \mathrm{x}$ \\
frisc & 2 & 479 & 1823 & 2364 & $912 \mathrm{x}$ & $5 \mathrm{x}$ \\
s38584 & 3 & 421 & 2305 & 2806 & $768 \mathrm{x}$ & $7 \mathrm{x}$ \\
s38417 & 4 & 709 & 3719 & 4463 & $930 \mathrm{x}$ & $6 \mathrm{x}$ \\
clma & 9 & 2735 & 53712 & 56726 & $5968 \mathrm{x}$ & $21 \mathrm{x}$ \\
\hline Ave & 1 & 335 & 3660 & 2775 & $3607 \mathrm{x}$ & $8 \mathrm{x}$ \\
\hline
\end{tabular}

Table 4: Runtime (second) comparison between EdTLC-LP and EdTLC-NW

\section{CONCLUSIONS}

To reduce power in dual-Vdd FPGA, we have re-formulated the LP based time slack allocation problem to a min-cost network flow based problem and presented a new network flow based algorithm, EdTLC-NW, with significantly shorter run-time. Using single-Vdd FPGA with power-gating as the baseline, the linear programming (LP) based time budgeting algorithm EdTLC-LP [12] and EdTLC-NW reduce interconnect power by $52.71 \%$ and $52.52 \%$, respectively. EdTLCNW achieves as good results as EdTLC-LP but runs $8 X$ faster on average. The speedup increases for larger circuits. For the largest circuit, EdTLC-NW achieves up to $6000 X$ speedup in time slack allocation and $20 X$ speedup in overall runtime. Clearly, the efficiency of EdTLC-NW makes our algorithm highly scalable, especially for the applications that may need iterative budgeting procedures. We expect that EdTLC-NW has more speedup in real designs than that reported in this paper since real designs are often bigger than the examples in this paper.

\section{REFERENCES}

[1] K. Poon, A. Yan, and S. Wilton, "A flexible power model for FPGAs," in Proc. of 12th International conference on Field-Programmable Logic and Applications, Sep 2002.

[2] F. Li, D. Chen, L. He, and J. Cong, "Architecture evaluation for power-efficient FPGAs," in Proc. ACM Intl. Symp. Field-Programmable Gate Arrays, Feb 2003. 


\begin{tabular}{|c|c|c|c|c|c|c|c|c|}
\hline \multirow[t]{3}{*}{1} & 2 & 3 & 4 & 5 & 6 & 7 & 8 & $\overline{9}$ \\
\hline & \multicolumn{2}{|c|}{ baseline power (W) } & \multicolumn{2}{|c|}{ interconnect power reduction } & \multicolumn{2}{|c|}{ dynamic power reduction } & \multicolumn{2}{|c|}{ leakage power reduction } \\
\hline & dynamic & leakage & EdTLC-LP & EdTLC-NW & EdTLC-LP & EdTLC-NW & EdTLC-LP & EdTLC-NW \\
\hline ex5p & 0.017687 & 0.000744 & $40.64 \%(3.25 \%)$ & $40.42 \%(1.31 \%)$ & $40.31 \%(3.28 \%)$ & $40.08 \%(1.22 \%)$ & $48.33 \%(2.55 \%)$ & $48.47 \%(3.47 \%)$ \\
\hline apex 4 & 0.020157 & 0.000768 & $45.24 \%(4.92 \%)$ & $44.19 \%(1.49 \%)$ & $44.91 \%(4.93 \%)$ & $43.87 \%(1.34 \%)$ & $53.79 \%(4.81 \%)$ & $52.38 \%(5.19 \%)$ \\
\hline misex 3 & 0.037557 & 0.000768 & $44.57 \%(3.23 \%)$ & $44.02 \%(2.73 \%)$ & $44.40 \%(3.23 \%)$ & $43.86 \%(2.65 \%)$ & $52.83 \%(2.89 \%)$ & $52.14 \%(6.70 \%)$ \\
\hline alu4 & 0.031968 & 0.000617 & $48.01 \%(6.09 \%)$ & $47.92 \%(1.55 \%)$ & $47.83 \%(6.14 \%)$ & $47.73 \%(1.50 \%)$ & $57.66 \%(3.34 \%)$ & $57.45 \%(4.15 \%)$ \\
\hline seq & 0.053091 & 0.001042 & $45.43 \%(3.58 \%)$ & $45.06 \%(1.65 \%)$ & $45.31 \%(3.59 \%)$ & $44.94 \%(1.60 \%)$ & $51.71 \%(2.96 \%)$ & $51.25 \%(3.95 \%)$ \\
\hline apex 2 & 0.053479 & 0.00121 & $49.71 \%(4.35 \%)$ & $49.56 \%(0.95 \%)$ & $49.51 \%(4.39 \%)$ & $49.36 \%(0.90 \%)$ & $58.65 \%(2.62 \%)$ & $58.33 \%(2.97 \%)$ \\
\hline des & 0.069708 & 0.001174 & $50.18 \%(1.31 \%)$ & $50.02 \%(0.74 \%)$ & $50.02 \%(1.30 \%)$ & $49.86 \%(0.73 \%)$ & $59.73 \%(1.78 \%)$ & $59.80 \%(1.78 \%)$ \\
\hline spla & 0.042551 & 0.002538 & $49.88 \%(3.74 \%)$ & $49.75 \%(1.37 \%)$ & $49.39 \%(3.80 \%)$ & $49.27 \%(1.24 \%)$ & $58.09 \%(2.65 \%)$ & $57.83 \%(3.68 \%)$ \\
\hline ex1010 & 0.028371 & 0.003003 & $46.08 \%(4.96 \%)$ & $45.89 \%(1.38 \%)$ & $44.74 \%(5.09 \%)$ & $44.53 \%(1.03 \%)$ & $58.77 \%(3.65 \%)$ & $58.76 \%(4.76 \%)$ \\
\hline $\mathrm{pdc}$ & 0.055369 & 0.003998 & $47.87 \%(3.42 \%)$ & $47.49 \%(1.08 \%)$ & $46.99 \%(3.53 \%)$ & $46.61 \%(0.97 \%)$ & $60.06 \%(1.97 \%)$ & $59.65 \%(2.71 \%)$ \\
\hline tseng & 0.006299 & 0.000545 & $62.39 \%(3.37 \%)$ & $62.39 \%(0.06 \%)$ & $61.44 \%(3.53 \%)$ & $61.45 \%(0.03 \%)$ & $73.29 \%(1.55 \%)$ & $73.34 \%(0.39 \%)$ \\
\hline dsip & 0.049421 & 0.000842 & $55.71 \%(0.97 \%)$ & $55.67 \%(0.72 \%)$ & $55.50 \%(0.97 \%)$ & $55.46 \%(0.71 \%)$ & $68.00 \%(0.97 \%)$ & $68.12 \%(1.38 \%)$ \\
\hline diffeq & 0.004697 & 0.000741 & $61.92 \%(2.93 \%)$ & $61.93 \%(0.14 \%)$ & $60.72 \%(3.13 \%)$ & $60.73 \%(0.05 \%)$ & $69.49 \%(1.64 \%)$ & $69.57 \%(0.68 \%)$ \\
\hline s298 & 0.010679 & 0.000884 & $57.06 \%(4.80 \%)$ & $57.04 \%(0.29 \%)$ & $56.26 \%(5.06 \%)$ & $56.23 \%(0.25 \%)$ & $66.74 \%(1.70 \%)$ & $66.86 \%(0.78 \%)$ \\
\hline bigkey & 0.049461 & 0.000956 & $48.14 \%(0.98 \%)$ & $47.84 \%(3.07 \%)$ & $47.90 \%(0.97 \%)$ & $47.60 \%(3.03 \%)$ & $60.68 \%(1.57 \%)$ & $60.54 \%(4.91 \%)$ \\
\hline elliptic & 0.015046 & 0.002065 & $62.71 \%(2.91 \%)$ & $62.72 \%(0.01 \%)$ & $61.04 \%(3.11 \%)$ & $61.04 \%(0.01 \%)$ & $74.94 \%(1.43 \%)$ & $74.95 \%(0.04 \%)$ \\
\hline frisc & 0.012426 & 0.002612 & $64.32 \%(3.78 \%)$ & $64.32 \%(0.01 \%)$ & $62.05 \%(4.18 \%)$ & $62.05 \%(0.00 \%)$ & $75.11 \%(1.87 \%)$ & $75.11 \%(0.05 \%)$ \\
\hline s38584.1 & 0.058038 & 0.002767 & $62.00 \%(1.69 \%)$ & $62.04 \%(0.09 \%)$ & $61.44 \%(1.71 \%)$ & $61.48 \%(0.09 \%)$ & $73.89 \%(1.35 \%)$ & $73.86 \%(0.25 \%)$ \\
\hline s38417 & 0.056849 & 0.003581 & $54.86 \%(1.98 \%)$ & $54.69 \%(1.47 \%)$ & $54.07 \%(1.99 \%)$ & $53.91 \%(1.43 \%)$ & $67.36 \%(1.79 \%)$ & $67.06 \%(2.15 \%)$ \\
\hline clma & 0.070958 & 0.008078 & $57.55 \%(4.70 \%)$ & $57.39 \%(0.50 \%)$ & $56.68 \%(5.06 \%)$ & $56.53 \%(0.41 \%)$ & $65.17 \%(1.51 \%)$ & $64.93 \%(1.32 \%)$ \\
\hline Ave. & 0.037191 & 0.001947 & $52.71 \%(3.35 \%)$ & $52.52 \%(1.03 \%)$ & $52.03 \%(3.45 \%)$ & $51.83 \%(0.96 \%)$ & $62.71 \%(2.23 \%)$ & $62.52 \%(2.57 \%)$ \\
\hline
\end{tabular}

Table 3: Total power achieved by EdTLC-LP and EdTLC-NW

[3] J. Lamoureux and S. J. Wilton, "On the interaction between power-aware FPGA CAD algorithms," in Proc. Intl. Conf. Computer-Aided Design, pp. 701-708, November 2003.

[4] J. H. Anderson, F. N. Najm, and T. Tuan, "Active leakage power optimization for FPGAs," in Proc. ACM Intl. Symp. Field-Programmable Gate Arrays, Februray 2004.

[5] F. Li, Y. Lin, L. He, and J. Cong, "Low-power FPGA using pre-defined dual-vdd/dual-vt fabrics," in Proc. ACM Intl. Symp. Field-Programmable Gate Arrays, Februray 2004

[6] F. Li, Y. Lin, and L. He, "FPGA power reduction using configurable dual-vdd," in Proc. Design Automation Conf., June 2004

[7] A. Gayasen, K. Lee, N. Vijaykrishnan, M. Kandemir, M. J. Irwin, and T. Tuan, "A dual-vdd low power FPGA architecture," in Proc. Intl. Conf. Field-Programmable Logic and its Application, August 2004.

[8] Fei Li and Yan Lin and Lei He, "Vdd programmability to reduce FPGA interconnect power," in Proc. Intl. Conf. Computer-Aided Design, November 2004.

[9] Jason H. Anderson and Farid N. Najm, "Low-power programmable routing circuitry for FPGAs," in Proc. Intl. Conf. Computer-Aided Design, November 2004.

[10] Y. Lin and L. He, "Leakage efficient chip-level dual-vdd assignment with time slack allocation for FPGA power reduction," in Proc. Design Automation Conf., June 2005.

[11] Y. Lin, F. Li, and L. He, "Power modeling and architecture evaluation for FPGA with novel circuits for vdd programmability," in Proc. ACM Intl. Symp. Field-Programmable Gate Arrays, Februray 2005.
[12] Y. Hu, Y. Lin, L. He, and T. Tuan, "Simultaneous time slack budgeting and retiming for dual-vdd FPGA power reduction," in Proc. Design Automation Conf., July 2006.

[13] V. Betz, J. Rose, and A. Marquardt, Architecture and CAD for Deep-Submicron FPGAs. Kluwer Academic Publishers, Feb 1999.

[14] S. C. Soheil Ghiasi, Elaheh Bozorgzadeh and M. Sarrafzadeh, "A unified theory of timing budget management," in Proc. Intl. Conf. Computer-Aided Design, November 2004.

[15] A. V. Goldberg, "An efficient implentation of a scaling minimum-cost flow algorithm," Journal of Algorithms, vol. 22, pp. 1-29, 1997.

[16] C. L. R. Rivest, T. Cormen, An Introduction to Algorithms. MIT Press, 1990.

[17] S. Yang, "Logic synthesis and optimization benchmarks, version 3.0," tech. rep., Microelectronics Center of North Carolina (MCNC), 1991.

[18] D. Lewis and et al, "The stratix routing and logic architecture," in Proc. ACM Intl. Symp. Field-Programmable Gate Arrays, Feb 2003.

[19] M Berkelaar, lp-solver: a public domain (MI)LP solver. ftp://ftp.ics.ele.tue.nl/pub/lp_solve/.

[20] "Xilinx product datasheets," in http://www.xilinx.com/literature. 